\title{
Factores metodológicos en la enseñanza-aprendizaje de los casos de factorización
}

\author{
Br. Mayquel José Monge, Bra. Hellen Manuela Orozco Dávila, \\ Bra. Isamar Gonzales Aguilar, Bra. Katherine Salguera Solano.
}

Facultad Regional Multidisciplinaria de Chontales. ${ }^{1}$

Recibido 24 de abril 2013-Aprobado 28 de octubre 2013.

\section{RESUMEN}

Este documento refleja la investigación interactiva con docentes y estudiantes de noveno grado del Instituto 21 de Junio de Santo Tomás Chontales. El propósito principal es apoyar metodológicamente la labor del docente afianzando y asegurando el aprendizaje de los estudiantes con relación al contenido de factorización. Este trabajo organizativo y participativo contiene conceptos y teorías que nos ayudan a comprender el problema, como surge y que nivel de transcendencia tiene en la época actual. Además, contiene una propuesta metodológica, respondiendo a las necesidades encontradas en el instituto donde se aplicaron los instrumentos. Se caracteriza la metodología implementada por el docente al enseñar Factorización, se describe el nivel de aprendizaje de cada uno de los estudiantes en cuestión de estudio y sugiere una propuesta metodología para mejorar el proceso de enseñanza aprendizaje.

Palabras clave: factorización, noveno grado, estrategias, metodología.

\section{INTRODUCCIÓN}

El presente trabajo investigativo tiene como finalidad hacer énfasis en la problemáticas que tienen los estudiante en el proceso de enseñanza-aprendizaje de los casos más comunes de factorización. Además, reflejamos las problemática encontrada en los estudiantes de la educación secundaria del noveno grado del Instituto 21 de junio de Santo Tomás.

La enseñanza de la matemática constituye la piedra angular para la formación y desarrollo de una conciencia lógica racional e intelectual del alumno. El proceso de enseñanza-aprendizaje de los casos más comunes de factorización constituye una experiencia positiva que sea marco de referencia para la complementación de este pensamiento lógico que demandan los tiempos, cambios y trasformaciones de la época. Caracterizar que las matemáticas y en nuestro caso, los casos más comunes de factorización y su aplicación para la vida práctica es un reto que debe realizarse en el proceso enseñanza-aprendizaje la cual constituirá un eje de motivación que despierte en los educando el estudio de esta importante asignatura. El rechazo de las matemáticas por los estudiantes es un problema muy complejo y las fallas se arrastran desde las escuelas. Se puede notar que existe una sucesión de errores en la concepción metodológica y la forma de percibir del educando y la forma de aplicación. Nuestra investigación está enmarcada en las líneas: proceso enseñanza-aprendizaje de los casos más comunes de factorización y que el docente uno de los principales participantes en este proceso está obligado a buscar el mejoramiento de los métodos de enseñanza.

\section{MATERIALES Y MÉTODOS}

Desde un enfoque cualitativo se estudiaron hechos, procesos y percepciones del problema en estudio, mediante un estudio de campo, para capturar los datos desde el interior. También se utilizó un enfoque cuantitativo que nos permitió emplear instrumentos y técnicas, que ayudaron a obtener datos concretos,

\footnotetext{
${ }^{1}$ Trabajo dirigido por el licenciado Jairo Flores.
} 
como es la encuesta y entrevistas. Los participantes de esta investigación son: Los docentes del área de matemática del noveno grado y los estudiantes del noveno grado de los turnos matutinos y vespertinos del Instituto 21 de Junio del municipio de Santo Tomás Chontales.

Las poblaciones en estudio son dos: los estudiantes del noveno grado del turno vespertino que totalizan 47 (18 niños y 29 niñas) y la segunda población es el docente de matemática. Todos ubicados en el Instituto 21 de Junio correspondiente al Municipio de Santo Tomas Chontales. Se tomó una muestra de 20 persona que representan $42 . \%$. El muestro utilizado fue el muestreo aleatorio simple que consiste en listar toda la población en estudio luego se selecciona la muestra según el tamaño de la misma, Por tanto, se seleccionó la muestra para ambos sexos atendiendo su representatividad en la población, en este particular la población está dividida en un $61 \%$ femenina y $39 \%$ masculina, por lo tanto se seleccionaron 12 mujeres y 8 varones de la forma antes detallada.

Criterios de inclusión de la muestra: Para la selección de los/as estudiantes se consideran los siguientes criterios: que estudien en el Instituto Nacional 21 de Junio; que estén en noveno grado de educación secundaria; que estudien en el turno vespertino. Para la selección de docentes se consideran los siguientes criterios: que sean maestros de educación secundaria; que impartan el área de matemática; que labore en Instituto Nacional 21 de Junio e imparta la asignatura de matemática en noveno grado; que hayan impartido matemáticas en noveno grado año de educación secundaria

Instrumentos y Técnicas: Las técnicas de recolección de información utilizadas para la recaudación de información y que son consideras las fuentes primarias son las siguientes:

Entrevistas estructuradas: con el fin de determinar cuáles son las dificultades que los docentes perciben en sus estudiantes al impartir los casos de factorización

Encuestas estructuradas: Dirigidas a estudiantes.

Examen diagnóstico: Se aplicó para explorar cuáles eran los conocimientos de factorización que poseen los estudiantes y si estos se encuentran acorde al nivel que estaban.

Observación directa: se logrará conocer, comprobar y recoger informaciones articulares y visuales de los estudiantes y docentes del tema estudiado.

Observaciones indirectas: permitió descubrir algunos factores que inciden en el aprendizaje de factorización.

El procesamiento de la información: Se realizó con el programa SPSS que permitió cuantificar la información de la encuesta. También de manera manual para el caso de las entrevistas, observaciones y examen diagnóstico, por último se utilizó los programas de Excel y Word para presentar la información con gráficas y de manera digital.

\section{RESULTADOS}

\section{Nivel de dominio de los casos de factorización por parte de los estudiantes}

El examen diagnóstico aplicado a los estudiantes de noveno grado fue una evaluación destinada a comprender la diversidad de métodos que el docente proporciona a los discentes sobre los casos de factorización estudiados. En el grafico 1, se puede observar que el $90 \%$ de los educandos mostró un deficiente nivel cognoscitivo de los casos de factorización (por debajo del 60) y tan solo el 10\% aprobó la prueba (en un rango de 61 a 62 ). 


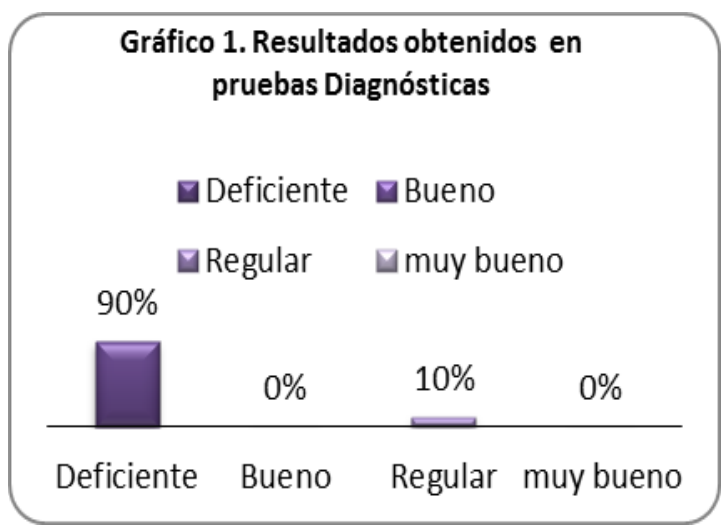

Esto conlleva a considerar que los conocimientos que deberían ser impartidos en noveno grado de educación secundaria quedaron a medias. En otras palabras, sin culminar y con poca profundidad. Primero, porque faltó tiempo y la aplicación de los contenidos a situaciones concretas de la vida; segundo, porque los docentes no lograron cumplir con el programa de estudio dejando pendiente los contenidos de factorización, y por consiguiente esto llevó a obtener un aprendizaje deficiente en los estudiantes con respecto a la factorización. Además, el docente en no proporcionar diversidad de métodos para la resolución de los casos de factorización a los discentes ha creado un rechazo y una apatía a la asignatura de matemática. Sobre todo al contenido de álgebra.

\section{Distribuciones de rendimiento por punto de prueba diagnóstica}

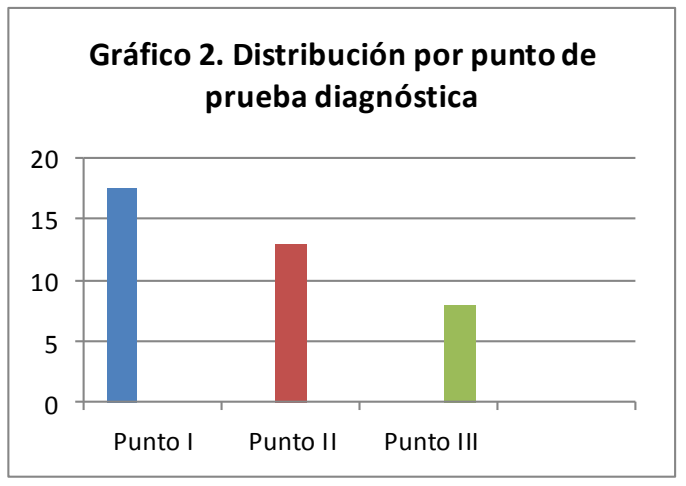

En el gráfico 2, se puede observar la separación del rendimiento promedio por los diferentes puntos de la prueba diagnóstica. El punto I aporta el $45.6 \%$ siendo el punto donde se tuvo mejor desempeño; el punto II aporta el 33.6\% del valor de la media siendo el segundo lugar en importancia. En este observamos el segundo nivel de enseñanza aprendizaje el saber lo cual nos indica de que esta en un aprendizaje de aprendizaje bajo., el punto III aporto un $20.8 \%$ demostrando que el nivel cognoscitivo del saber hacer del discente es deficiente. Es probable que estos resultados se deban a que el punto uno sea el que exige menos habilidades matemáticas que los ejercicios del punto III en donde se requiere que el estudiante logre abstraer la problemática y la resolviera correctamente, esta habilidad se forma por medio de un enfoque de enseñanza basado en la resolución de problemas y no bajo el sistema tradicional de enseñanza mecánica de la matemática.

\section{Porcentaje de dominio que obtuvieron los estudiantes en cada caso de factorización.}

Fue evidente que el caso más fácil de resolver es el caso factor común, el cual obtuvo un $65 \%$ de aprobación, seguido del trinomio cuadrado perfecto. Los casos que más se les dificultó fueron: el caso del trinomio de la forma $a x^{2}+b x+c$ con un $30 \%$ de dominio y el trinomio de la forma $\mathrm{x}^{2}+\mathrm{bx}+\mathrm{c}$ con un 35\% de dominio de los estudiantes. Esto muestra que la hipótesis planteada en el problema de investigación lo obtienen los casos 6 y 7 de factorización ya que de acuerdo a lo observado la limitación de diversidad de métodos para resolverlo dificulta el nivel de enseñanza aprendizaje de los discentes

\section{Factores que influyeron en la enseñanza y aprendizaje de los casos de factorización}

El principal factor que intervino en el desarrollo del contenido factorización en noveno grado fue la programación del contenido. Este se tenía que desarrollar a finales del mes de abril, según el programa educativo. Pero el docente expresó que la realidad era otra, el contenido se desarrolló hasta el mes de junio del 2012. Por lo que los estudiantes se vieron afectados ya que no se les enseñó en el tiempo predestinado para cada caso de factorización. Otro agente o factor fue la presentación del contenido. Mediante las observaciones y entrevistas aplicadas al docente se descubrió que los casos de factorización 
dados a los estudiantes no tuvieron gran significado puesto que dichas actividades de enseñanza aprendizaje no mostraban su aplicación a la vida, además de que se sigue recurriendo a una metodología de enseñanza tradicionalista que se transmite de generación en generación provocando el poco interés y la indisciplina de los mismos.

\section{Dificultades que enfrenta el docente para la enseñanza de factorización.}

1. Aulas de clase saturadas de estudiantes desarrolla su clase con un promedio de 47 estudiantes, cuando lo recomendable es 25 estudiantes por tanto se le dificulta brindar atención individual a todos los estudiantes.

2. Estudiantes con diferentes edades y niveles de aprendizaje.

3. La poca bibliografía de lo único que se apoya es del álgebra Baldor careciendo así de diversidad de aprendizajes.

4. El apoyo del jefe de área, es únicamente para la revisión de planes diarios.

5. Distractores en el aula de clase (celulares, computadoras, radios).

6. Escasez de recursos y medios didácticos.

7. Desinterés estudiantil.

8. Indisciplina escolar.

9. No existe ningún asesoramiento pedagógico.

10. Alto índice de inasistencia e impuntualidad a las clases.

11. Bibliotecas que carecen de documentos y material matemático.

\section{CONCLUSIONES}

Los principales factores que influyen en el proceso de enseñanza-aprendizaje en los casos más comunes de factorización son el desinterés del educando y la poca innovación de estrategias por parte del docente al desarrollar un contenido dentro del aula de clase.

El nivel general de conocimiento de los educandos tiene un índice muy bajo, teniendo mayor debilidad en los casos seis y siete de factorización.

En torno a la hipótesis planteada, se verifica como factor principal la metodología implementada por el docente que es poco innovadora y el grado de interés de los educandos es bajo lo que dificulta que el proceso de enseñanza de los casos más comunes de factorización se logren desarrollar de una manera significativa en los educandos

\section{BIBLIOGRAFÍA}

Morales E, (2010). ¿Cómo identificar los casos de factorización? (en línea).Consultado mayo 2012(en línea). Disponible en: http://www.articuloz.com/escuela-en-casa-articulos/como-identificar-loscasos-de factorizacion-2247651.html

Martha T. Gutiérrez (2006). Módulo de pedagogía general. Managua, Nicaragua. Primera impresión de 800 ejemplares.

Velázquez, Martha; De León, Alejandro; Mendoza, Raúl (2009). Coordinación Educativa y Cultural Centroamericana Colección Pedagógica Formación de Docentes Centroamericanos de educación Primaria Básica. San José, Costa Rica: Impresión Litográfica Editorama S.A.

Woolfolk, Anita E. (1999). Psicología Educativa. México: Pretince Hail hispanoamericana S.A. 